\title{
Integrative Aspekte der Thoraxonkologie
}

\author{
Markus Horneber $^{\mathrm{a} *}$ Anja Mehnert ${ }^{\mathrm{b} *}$ Traugott Roser $^{\mathrm{c} *} \quad$ Matthias Rostock $^{\mathrm{d}, \mathrm{e} *}$ \\ ${ }^{a}$ Medizinische Klinik 5, Zentrum für Onkologie, Hämatologie, Stammzelltransplantation, Infektiologie und Palliativmedizin, \\ Klinikum Nürnberg, \\ b Institut und Poliklinik für Medizinische Psychologie, Universitätsklinikum Hamburg-Eppendorf, \\ ${ }^{c}$ Professur für Spiritual Care, Interdisziplinäres Zentrum für Palliativmedizin, Klinikum der Universität München, \\ Campus Großhadern, München, Deutschland \\ ${ }^{d}$ Institut für Naturheilkunde, Universitätsspital Zürich, Schweiz \\ e Universitäres Cancer Center Hamburg, Hubertus Wald Tumorzentrum, Universitätsklinikum Hamburg-Eppendorf, Deutschland
}

\section{Ansätze in der ganzheitlichen Behandlung und}

Betreuung krebskranker Menschen und deren Evidenz

Die moderne Onkologie kennzeichnet ein naturwissenschaftlich geprägtes Krankheitsmodell und krankheitszentriertes Vorgehen in Diagnostik und Therapie. Ganzheitliche Ansätze versuchen, natur- und humanwissenschaftliche Medizin im Sinne einer integrativen Onkologie zusammenzuführen und Patienten nicht auf ihre Krankheiten zu reduzieren, sondern in ihrer Ganzheit und unter Berücksichtigung der individuellen Lebenskontexte nach medizinischen, pflegerischen, psychosozialen und spirituellen Aspekten umfassend zu versorgen. Solche Ansätze erfordern nicht nur einen intensiven und interdisziplinären Diskurs über das zugrunde liegende Verständnis von Gesundheit und Krankheit, sondern auch eine Verständigung darüber, wie Wissen, therapeutische Erfahrung und die Bedürfnisse der Patienten bei klinischen Entscheidungen sinnvoll und zum Nutzen der Betroffenen integriert werden können [1].

Für die Versorgung von Patienten mit Lungenkarzinomen gibt beispielsweise die Interdisziplinäre S3-Leitlinie durchaus in diesem Sinne integrative Strukturen vor, indem sie die Vorstellung in einem interdisziplinären Zentrum unter Einbeziehung von Pneumologie, internistischer Onkologie, Radioonkologie, Thoraxchirurgie, Ernährungsberatung und -therapie, psychologischer Beratung und Betreuung, Sozialdienst und Palliativmedizin empfiehlt [2].

Auf der Ebene der individuellen Patientenversorgung treffen ganzheitliche, integrative Konzepte bereits auf die Schwierigkeit, die verschiedenen Perspektiven der Fachleute

*Alle Autoren haben zu gleichen Teilen zu diesem Artikel beigetragen. untereinander und mit der individuellen Wirklichkeit des Kranken und seines Umfelds zu vermitteln. Es bedarf eines andauernden Bemühens aller Beteiligten, die sich im Verlauf der Krankheit und der Behandlung ändernden Bedürfnisse, Anforderungen und Notwendigkeiten in ein gemeinsames «Ganzes» als Grundlage des Behandlungsbündnisses zu integrieren. Dies kann deutlich werden aus Untersuchungen, die zeigen, wie sehr sich Fachleute, Angehörige und Patienten in ihrer jeweiligen Sicht der individuellen Krankheitssituation unterscheiden und wie stark und häufig unerwartet sich das Bedürfnis nach Information und Teilhabe an Entscheidungsprozessen während des Krankheitsverlaufs ändert [3, 4].

Patienten und Angehörige verbinden mit einer ganzheitlichen Betreuung häufig die Ergänzung der «schulmedizinischen» Behandlung durch «komplementärmedizinische» Therapien. Nur wenige Studien untersuchten die Wirkungen derartiger «ganzheitlicher» Konzepte bei Lungenkarzinompatienten. In einer retrospektiven Kohortenstudie fanden McCulloch et al. [5] beispielsweise deutlich verbesserte Überlebensraten in der Gruppe der Patienten, die während einer konventionellen Chemo- und Strahlentherapie mit einem «Whole Systems Approach» nach Aspekten der Traditionellen Chinesischen Medizin (TCM) behandelt worden waren. Zusätzlich zu der sehr eingeschränkten Evidenz dieser Ergebnisse sollte bei der Integration der TCM in die westliche Medizin unter dem Aspekt der Ganzheitlichkeit berücksichtigt werden, dass «Traditional Chinese Medicine (...) has its own holism and individualization» [6].

Als ein gelungener Ansatz einer ganzheitlichen Betreuung und Behandlung von Patienten mit neu diagnostiziertem, metastasiertem nichtkleinzelligen Lungenkarzinom (NSCLC) kann die Phase-III-Studie von Temel et al. [7] betrachtet werden. In dieser führte ein integriertes onkologisch-palliativme-

\begin{tabular}{ll}
\hline KARGER & ( ) 2012 S. Karger GmbH, Freiburg \\
0378-584X/12/3515-0002\$38.00/0 \\
$\begin{array}{l}\text { Fax+49 7614520714 } \\
\text { Information@Karger.de } \\
\text { www.karger.com }\end{array}$ & $\begin{array}{l}\text { Accessible online at: } \\
\text { www.karger.com/onk }\end{array}$
\end{tabular}


dizinisches Behandlungskonzept, in dem medizinische, pflegerische, psychologische, soziale und spirituelle Aspekte im oben genannten Sinne integriert wurden, bei den Betroffenen $\mathrm{zu}$ besserer Lebensqualität, verringerter psychischer Belastung und einem verlängerten Überleben trotz geringeren Einsatzes aggressiver Therapien am Lebensende.

\section{Welchen Beitrag kann Spiritualität leisten?}

Die Integration spiritueller Bedürfnisse in die Therapie unheilbar kranker Menschen ist ein wesentliches Merkmal einer ganzheitlichen palliativmedizinischen Versorgung, die nach der Definition der Weltgesundheitsorganisation (WHO) unter anderem die Anamnese, Diagnose und Therapie physischer, psychosozialer und spiritueller Probleme umfasst [8]. Dabei handelt es sich nicht um klar abgrenzbare Dimensionen; die Bereiche können sich vielmehr überschneiden, wobei sich die Spiritualität des Einzelnen von derjenigen der Betreuer deutlich unterscheiden kann. Nach einer vorläufigen Arbeitsdefinition der European Association for Palliative Care (EAPC) Task Force umfasst Spiritualität Aspekte einer existenziellen Herausforderung, Wert- und Weltanschauungsfragen sowie religiöse und glaubensbezogene Ansichten [9].

Da Spiritualität letztlich nicht eindeutig bestimmbar und verbindlich definierbar ist, sollte sich Spiritual Care weniger an der vermeintlich objektiven Wirksamkeit spiritueller Praktiken orientieren, als vielmehr an den spirituellen Ressourcen des individuellen Patienten, seiner spirituellen Situation sowie seinen spirituellen Nöten und Bedürfnissen ansetzen. Dabei verdeutlicht eine in Deutschland durchgeführte Untersuchung von Patienten mit chronischen Erkrankungen, dass die Bedürfnisse nach innerem Frieden und nach Weitergabe (von Lebenserfahrungen, Trost usw.) für die Bewertung der Krankheit von größerer Relevanz sind als explizit religiöse oder existenzielle Bedürfnisse [10].

Als wichtigste «Anbieter» von Spiritual Care identifizierte eine US-amerikanische Studie vor allem Familie und Freunde (41\%) sowie Ärzte und Krankenschwestern (29\%), Seelsorger rangierten mit einem Anteil von 17\% dagegen erst an 3. Stelle [11]. Dies unterstreicht zum einen die große Bedeutung des sozialen Umfeldes im spirituellen Umgang mit der Krankheit, impliziert aber auch die Frage, wie Spiritual Care in den Behandlungsplan integriert werden kann.

Nach einem in der Professur für Spiritual Care an der LMU München entwickelten Modell sollte zunächst eine auf 5-10 min begrenzte spirituelle Anamnese mit Dokumentation erfolgen. Das daraufhin hinzugezogene interdisziplinäre Team untersucht dann, wie Spiritual Care in den Gesamtbehandlungsplan aufgenommen und die spirituellen Bedürfnisse des Patienten erfüllt sowie seine entsprechenden Ressourcen am besten genutzt werden können (Abb. 1).

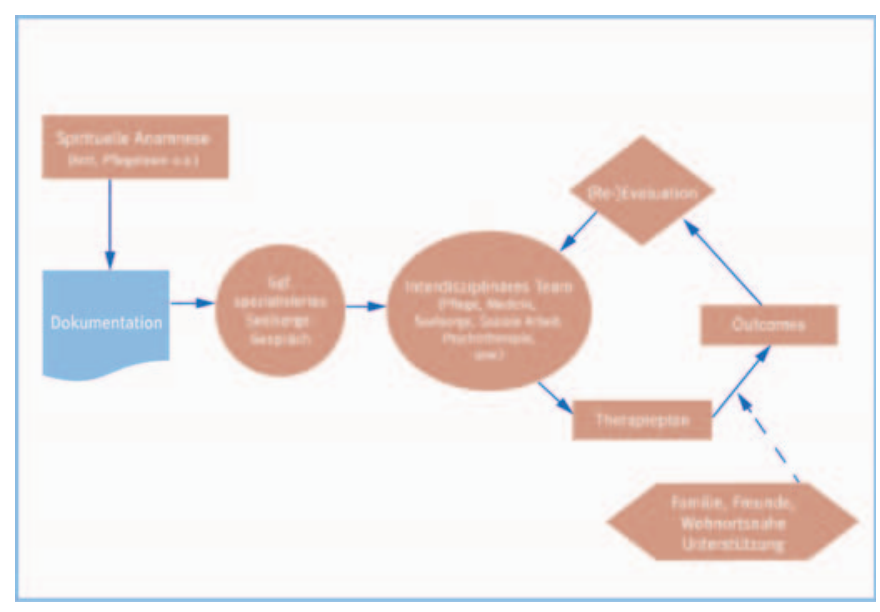

Abb. 1. Integration von Spiritual Care in den Behandlungsplan $\left({ }^{\circ}\right.$ E. Frick, T. Roser, 2011).

\section{Komplementärmedizinische Methoden}

Viele Tumorpatienten wenden im Krankheitsverlauf komplementärmedizinische Methoden an, um krankheitsbedingte Symptome und/oder Beschwerden durch ihre tumorspezifischen Therapien zu verringern und die Lebensqualität $\mathrm{zu}$ verbessern, zum Teil auch mit der Hoffnung, hierdurch die Prognose ihrer Erkrankung mit beeinflussen zu können. In diesem Kontext kann nur mit einigen Beispielen ein kleiner Einblick in das Spektrum häufiger angewandter komplementärmedizinischer Therapiemaßnahmen gegeben werden.

\section{Sport und Bewegung}

Ein Benefit körperlicher Aktivitäten konnte bei Patienten mit verschiedenen Tumorentitäten, besonders beim Mammakarzinom und kolorektalen Karzinom, durch kontrollierte Studien belegt werden. Einer großen Metaanalyse zufolge haben sportliche Aktivitäten während und nach einer AntitumorTherapie große Effekte auf die Körperkraft, moderate Effekte auf Fatigue sowie geringe bis mäßige Effekte auf den körperlichen Aktivitätslevel, Fitness, Lebensqualität, Angst und Selbstwertgefühl [12].

\section{Vitamine, Mineralstoffe und Spurenelemente}

Weniger konsistent ist die Datenlage zum Einsatz von Vitaminen, Mineralstoffen und Spurenelementen. Während der Einsatz von Antioxidanzien begleitend zu einer Chemo- und/ oder Strahlentherapie oft kritisch beurteilt wird, weil eine mögliche Wirkungseinschränkung der antitumoralen Behandlung befürchtet wird [13], hatten NSCLC-Patienten in einer an der Mayo-Klinik durchgeführten Kohortenstudie bei Einnahme von Vitaminen und Mineralstoffen einen statistisch signifikanten Überlebensvorteil im Vergleich zu Patienten ohne entsprechende Supplementierung (Hazard Ratio (HR) 0,74; 95\%-Konfidenzintervall (KI) $0,60-0,91 ; \mathrm{p}<0,01$ ) [14]. 
Da sehr viele Patienten Vitamin- und Mineralstoffpräparate begleitend $\mathrm{zu}$ ihrer antitumoralen Behandlung einsetzen, wären kontrollierte klinische Studien sehr wünschenswert, angesichts der ubiquitären Erhältlichkeit der Präparate als sogenannte Nahrungsergänzungsmittel und fehlender öffentlicher Forschungsförderung allerdings nur schwer durchführbar.

\section{Phytotherapie}

Zur Sicherheit und Wirksamkeit von Mistelextrakten liegen die Ergebnisse einer Cochrane-Analyse von 21 prospektiven, randomisierten Studien, darunter 2 bei Patienten mit NSCLC, vor. Die Arzneimittelsicherheit wurde in 12 Studien untersucht, die alle eine gute Verträglichkeit zeigten. Auf einen Überlebensvorteil deuteten 6 von 13 Studien, wobei allerdings keine dieser Studien eine höhere methodische Qualität aufwies. 7 Studien, darunter 4 mit höherer methodischer Qualität, zeigten keinen Nutzen. Für eine positive Bewertung der antitumoralen Wirksamkeit reichten die Daten somit nicht aus. Eine Verbesserung von zumindest Teilaspekten der Lebensqualität und/oder Verträglichkeit von Chemo- oder Strahlentherapie unter begleitender Misteltherapie war dagegen in 14 von 16 Studien, darunter 2 mit höherer methodischer Qualität zu sehen [15].

Häufiges Ziel einer begleitenden Phytotherapie ist eine Beeinflussung von Nebenwirkungen der Chemotherapie. Patienten mit erhöhten Transaminasen könnten z.B. von Mariendistelextrakten profitieren. Silymarin und der Hauptwirkstoff Silibinin werden in letzter Zeit bei verschiedenen Tumorentitäten intensiv untersucht. In einer 2010 publizierten Übersichtsarbeit wurden die bisher für Silibinin vorliegenden Daten hinsichtlich verschiedener antitumoraler Effekte aus präklinischen und klinischen Studien zusammengefasst [16].

Für das Gummiharz des indischen Weihrauchbaums wurden neben antiphlogistischen ebenfalls verschiedene antitumorale Wirkungen wie Hemmung der Topoisomerase I und II und Apoptose-Induktion gezeigt. Die Wirksamkeit eines Weihrauchextrakts bei Patienten mit bestrahlten Hirntumoren (Glioblastom oder zerebrale Metastasen; die meisten in dieser Studie eingeschlossenen Patienten litten unter Hirnmetastasen bei NSCLC oder kleinzelligem Lungenkarzinom (SCLC)) wurde erstmals in Freiburg i.Br. in einer randomisierten, placebokontrollierten Studie untersucht. Bei den mit einem Weihrauchextrakt (4200 mg/Tag) behandelten Patienten nahm das Volumen des zerebralen Ödems (primärer Endpunkt) signifikant stärker $\mathrm{ab}$ als in der Placebogruppe ( $\mathrm{p}=$ 0,023). Das Volumen der größten Tumorläsion verringerte sich unter Einnahme des Weihrauchextrakts ebenfalls signifikant stärker als unter Placebo ( $\mathrm{p}=0,008)$, die nach RECISTKriterien bestimmte Tumor-Response war aber nicht signifikant unterschiedlich. Die Ergebnisse deuten auf einen möglichen Corticoid einsparenden Effekt hin, der aber, ebenso wie der Hinweis auf eine mögliche zusätzliche antitumorale Wirksamkeit, weiter evaluiert werden muss [17].
Die wissenschaftliche Datenlage zur Einschätzung der Evidenz komplementärmedizinischer Therapiemaßnahmen bei Patienten mit Bronchialkarzinomen ist nach wie vor unbefriedigend, bietet aber doch einige Hinweise auf möglicherweise sinnvolle Behandlungsansätze, die weiter verfolgt werden sollten.

\section{Psychosoziale Betreuung und Möglichkeiten der Unterstützung}

Eine Tumorerkrankung bedeutet für den Betroffenen einen tiefen Lebenseinschnitt, der eine existenzielle Belastung darstellen und mit starken Ängsten vor einer Progredienz der Erkrankung einhergehen kann. Besonders häufig finden sich diese bei Patienten mit Lungenkarzinom, wie unter anderem eine US-amerikanische Studie zeigte, in der $43 \%$ der Lungenkarzinom-Patienten unter hoher psychischer Belastung, wie Depressivität und Ängstlichkeit litten [18]. Die Bedürfnisse vieler Patienten und ihrer Angehörigen beziehen sich bei fortgeschrittener Erkrankung aber nicht allein auf die Kontrolle belastender Symptome, sondern auch auf die Unterstützung bei der Suche nach Hoffnung, Lebenssinn und innerem Frieden.

Wichtige Ziele der psychoonkologischen Unterstützung sind daher die Stärkung des Selbstwertgefühls und die Anerkennung der Lebensleistung des Patienten, die Verringerung von Gefühlen der Isolation und Einsamkeit, die Stärkung oder Klärung familiärer Bindungen, die Unterstützung adaptiver Bewältigungsstrategien, die Mobilisierung eigener Ressourcen, das Aufzeigen neuer Lebensperspektiven einschließlich neuer Hoffnungen sowie die Signalisierung von Offenheit gegenüber Aspekten, wie Trennung, Verlust, Tod oder Angst vor dem Unbekannten. Dabei werden neben psychotherapeutischen Interventionen, die bei körperlich Gesunden entwickelt wurden, auch Interventionen angeboten, die einen Lebensrückblick oder die Hinterlassenschaft für die nächste Generation ermöglichen.

\section{Hoffnung bei fortgeschrittener Tumorerkrankung}

Hoffnung und das Erleben von Sinnhaftigkeit können wesentlich zur Akzeptanz einer Erkrankung sowie zur Verbesserung des emotionalen Wohlbefindens und der Lebensqualität beitragen. Für viele unheilbar kranke Patienten ist es daher hilfreich, wenn der Psychoonkologe mit ihnen Alternativen zur Hoffnung auf Heilung erarbeitet:

- Hoffnung auf Symptomkontrolle

- Hoffnung auf Lebensqualität

- Hoffnung auf Begegnungen mit anderen Menschen

- Hoffnung, Liebe zu schenken und Liebe zu erfahren

- Hoffnung, dem Leben Sinn zu geben

- Hoffnung auf neue Erfahrungen, auf Reisen, auf fremde Kulturen

- Hoffnung auf Aussöhnung oder Klärung von Beziehungen 
- Hoffnung auf Vergebung

- Hoffnung auf ein persönliches Vermächtnis

- Hoffnung auf Akzeptanz der Endlichkeit des eigenen Lebens.

\section{Disclosure Statement}

Die Autoren waren Referenten beim «Dresdner Interdisziplinäres Gespräch 2012», das von Roche Pharma AG unterstützt wurde.

\section{Literatur}

1 National Research Council: Integrative Medicine and the Health of the Public: A Summary of the February 2009 Summit. Washington, DC, The National Academies Press, 2009, www.iom.edu/Reports/2009/Integrative-Medicine-Health-Public aspx.

2 Goeckenjan G, Sitter H, Thomas M, et al.: Prävention, Diagnostik, Therapie und Nachsorge des Lungenkarzinoms. Kurzfassung der Interdisziplinären S3-Leitlinie der Deutschen Gesellschaft für Beatmungsmedizin und der Deutschen Krebsgesellschaft. Pneumologie 2011;65:e51-e75.

3 Faller H, Lang H, Schilling S, Wagner J: Krankheitsverarbeitung bei Bronchialkarzinom aus Sicht der Patienten, ihrer Angehörigen und der Betreuer. Eine Fünf-Ebenen-Untersuchung. Psychother Psychosom Med Psychol 1992;42:322-331.

4 Pardon K, Deschepper R, Vander Stichele R, et al.: Changing preferences for information and participation in the last phase of life: a longitudinal study among newly diagnosed advanced lung cancer patients. Support Care Cancer 2012; DOI: $10.1007 /$ s00520-011-1369-4.

$>5$ McCulloch M, Broffman M, van der Laan M, et al.: Lung cancer survival with herbal medicine and vitamins in a whole-systems approach: Ten-year follow-up data analyzed with marginal structural models and propensity score methods. Integr Cancer Ther 2011;10:260-279.
6 Jeong SJ, Koh W, Kim B, et al.: Are there new therapeutic options for treating lung cancer based on herbal medicines and their metabolites? J Ethnopharmacol 2011;138:652-661.

7 Temel JS, Greer JA, Muzikansky A, et al.: Early palliative care for patients with metastatic nonsmall-cell lung cancer. N Engl J Med 2010;363:733742.

8 www.who.int/cancer/palliative/definition/en/

9 Nolan S, Saltmarsh P, Leget C: Spiritual care in palliative care - working towards an EAPC Task Force. Eur J Pall Care 2011;18:86-89.

10 Büssing A, Janko A, Kopf A, et al.: Zusammenhänge zwischen psychosozialen und spirituellen Bedürfnissen und Bewertung von Krankheit bei Patienten mit chronischen Erkrankungen. Spiritual Care 2012;1:57-73.

11 Hanson LC, Dobbs D, Usher BM, et al.: Providers and types of spiritual care during serious illness. J Palliat Med 2008;11:907-914.

12 Speck RM, Courneya KS, Mâsse LC, et al.: An update of controlled physical activity trials in cancer survivors: a systematic review and meta-analysis. J Cancer Surviv 2010;4:87-100.
13 Lawenda BD, Kelly KM, Ladas EJ, et al.: Should supplemental antioxidant administration be avoided during chemotherapy and radiation therapy? J Natl Cancer Inst 2008;100:773-783.

14 Jatoi A, Williams B, Nichols F, et al.: Is voluntary vitamin and mineral supplementation associated with better outcome in non-small cell lung cancer patients? Results from the Mayo Clinic lung cancer cohort. Lung Cancer 2005;49:77-84.

15 Horneber M, Bueschel G, Huber R, et al.: Cochrane Database Syst Rev 2008;(2):CD003297.

16 Cheung CW, Gibbons N, Johnson DW, et al.: Silibinin - a promising new treatment for cancer. Anticancer Agents Med Chem 2010;10:186-195.

17 Kirste S, Treier M, Wehrle SJ, et al.: Boswellia serrata acts on cerebral edema in patients irradiated for brain tumors: a prospective, randomized, placebo-controlled, double-blind pilot trial. Cancer 2011;117:3788-3795.

18 Zabora J, Brintzenhofe-Szoc K, Curbow B, et al. The prevalence of psychological distress by cancer site. Psychooncology 2001;10:19-28. 